\title{
Myofascial Massage for Chronic Pain and Decreased Upper Extremity Mobility After Breast Cancer Surgery
}

\author{
Jeanne Massingill, LMT, MLD, CST, KT, NMT, ${ }^{1}$ Cara Jorgensen, LMT, ${ }^{1}$ \\ Jacqueline Dolata, MBA, ${ }^{2}$ Ashwini R. Sehgal, MD ${ }^{2,3}$ \\ ${ }^{1}$ The Oaks Massotherapy and Botanical Spa, Rocky River, OH, ${ }^{2}$ Center for Reducing Health Disparities, Case Western
Reserve University, Cleveland, OH, ${ }^{3}$ Department of Internal Medicine, MetroHealth Medical Center, Cleveland, OH, USA
}

Background: Chronic localized pain and decreased upper extremity mobility commonly occur following breast cancer surgery and may persist despite use of pain medication and physical therapy.

Purpose: We sought to determine the value of myofascial massage to address these pain and mobility limitations.

Setting: The study took place at a clinical massage spa in the U.S. Midwest. The research was overseen by MetroHealth Medical Center's Institutional Review Board and Case Center for Reducing Health Disparities research staff.

Participants: 21 women with persistent pain and mobility limitations 3-18 months following breast surgery.

Research Design: We conducted a pilot randomized controlled trial where intervention patients received myofascial massages and control patients received relaxation massages.

Intervention: Intervention participants received 16 myofascial massage sessions over eight weeks that focused on the affected breast, chest, and shoulder areas. Control participants received 16 relaxation massage sessions over eight weeks that avoided the affected breast, chest, and shoulder areas. Participants completed a validated questionnaire at the beginning and end of the study that asked about pain, mobility, and quality of life.

Main Outcome Measures: Outcome measures include change in self-reported pain, self-reported mobility, and three quality-of-life questions.

Results: At baseline, intervention and control participants were similar in demographic and medical characteristics, pain and mobility ratings, and quality of life. Compared to control participants, intervention participants had more favorable changes in pain $(-10.7$ vs. $+0.4, p<.001)$, mobility $(-14.5$ vs. $-0.8, p<.001)$, and general health $(+29.5$ vs. $-2.5, p=.002$ ) after eight weeks. All intervention and control participants reported that receiving massage treatments was a positive experience.

Conclusions: Myofascial massage is a promising treatment to address chronic pain and mobility limitations following breast cancer surgery. Further work in several areas is needed to confirm and expand on our study findings.

KEY WORDS: massage, myofascial, breast cancer, chronic pain, decreased mobility

\section{INTRODUCTION}

About $25 \%-50 \%$ of women have chronic localized pain and decreased upper extremity mobility following breast cancer surgery. ${ }^{(1,2,3)}$ Breast cancer surgery may include biopsy, lumpectomy, mastectomy, or some type of reconstruction. ${ }^{(3)}$ Current treatments for these problems include pain medication and physical therapy, but such approaches are not always effective. ${ }^{(4)}$ Myofascial massage has been shown to be an effective treatment for other post-surgical patients in reducing pain and increasing mobility-for example, for patients undergoing various bladder surgeries after cancer. $^{(5)}$ Myofascial massage is a technique that focuses on applying fascial holds, stretching, stroking, and varied pressure to tissue at modified depths. However, a targeted myofascial massage technique geared towards the breast/chest/shoulder region has not been rigorously evaluated. ${ }^{(6)}$ This targeted myofascial massage also incorporates lymphatic drainage to achieve reduction of swelling in the arms and axilla area. ${ }^{(5)}$

We sought to determine the effectiveness of myofascial massage delivered to the breast/chest/shoulder area in addressing pain and mobility limitations among patients who underwent breast cancer surgery. To better understand the specific effects of myofascial massage, we included a randomly chosen control group that received an alternative relaxation massage that avoided the affected breast, chest, and shoulder areas. Relaxation massage techniques include Swedish relaxation massage and light touch, and have been used as a way of decreasing stress, anxiety, and some self-reported pain in the general population. ${ }^{(7)}$ If proven effective, myofascial massage could be an inexpensive and non-invasive addition to current pain and mobility treatments. ${ }^{(6,8)}$ 


\section{METHODS}

\section{Setting and Participants}

This pilot randomized controlled trial took place at a free-standing clinical massage spa in the U.S. Midwest. The study was approved by the MetroHealth Medical Center Institutional Review Board (IRB) before recruitment and enrollment began. Eligible participants were women age 18 years or older who had undergone breast cancer surgery 3-18 months prior to the study, and who had persistent pain or lack of mobility in their breast, chest, or shoulder area. Studies have varied on optimal time to begin any type of physiotherapy or massage, with some beginning as early as the next day following surgery for certain massage treatments. ${ }^{(8)}$ However, the timeline of 3-18 months was determined by the massage therapist and a consulting breast surgeon, based on clinical experience of tissue being overly sensitive before three months' time and less pliable after 18 month's time. The types of breast cancer surgery included biopsy, lumpectomy, mastectomy, or some type of reconstruction.

Study inclusion criteria focused on the level of pain and mobility limitations because our clinical experience indicated that the exact type of surgery is less relevant in determining response to massage. We excluded women if they had infected skin or open wounds, were currently receiving massage therapy, or were mentally incompetent as determined by the study coordinator based on ability to understand the study and consent form as required by the MetroHealth IRB. Women were recruited by distributing flyers through northeast Ohio breast surgeons' practices and cancer survivor organizations.

\section{Screening}

Interested women called the study coordinator and were screened by phone using reliable and valid questionnaires to determine if they met minimum pain and lack of mobility thresholds. $(9,10)$ To be eligible, women had to report mild pain on at least 2 items, moderate pain on at least 1 item, mild difficulty with at least 2 activities, or moderate difficulty with at least 1 activity. To exclude individuals who might not be able to adhere to study requirements, eligible women were then instructed to call the Oaks Botanical Spa to schedule their first appointment. Women who failed to come to their first appointment were excluded.

\section{Baseline Measures}

The two surveys used as screening tools also served as the baseline pain and mobility measurements. We utilized 10 pain items from the McGill Pain Questionnaire, a valid and reliable tool that asks individuals to describe their pain (e.g., throbbing or shooting) and then rate the severity of pain on a 4-point Likert scale (none, mild, moderate, severe). ${ }^{(9)}$ We also utilized a valid and reliable survey developed by Wingate involving 10 mobility items specifically developed for post-breast surgery patients that ask individuals to rate difficulty performing specific activities (e.g., brushing hair or putting on a T-shirt) on a 5-point Likert scale (no difficulty, mild difficulty, moderate difficulty, severe difficulty, unable to do). ${ }^{(10)}$ In addition to the pain and mobility questionnaires, women who met screening criteria were also asked three questions from the Short Form Health Survey (SF-12) about social activities, feeling downhearted, and overall health. ${ }^{(11,12)}$ Each of these surveys was administered one time over the phone in order to complete the baseline assessment. Women were also asked demographic and surgery-related information, including age, time post-surgery, type of surgery, whether they received radiation treatment, and whether they had seen a physical therapist or pain management specialist for their pain and mobility limitations.

\section{Randomization}

At the first appointment, participants received additional information about the trial and provided written informed consent. Envelopes for randomization were prepared and sealed in advance by the study coordinator. Each envelope contained four individually sealed slips of paper, two with the word "intervention" (myofascial) and two with the word "control" (relaxation) written on them. A licensed massage therapist (JM or CJ) then selected one of these sealed envelopes and withdrew a sealed slip from the envelope, thereby randomizing participants into intervention or control groups until the four slips of paper were used up. In this way, intervention and control patients were more evenly spread out throughout the several months of the pilot. Based on the voluntary nature of the massage therapists' time, this pilot study sought to enroll about 20 patients (10 in each group) in order to examine potential efficacy.

\section{Intervention}

Both control and intervention groups received two 30-minute massages per week for eight weeks (total of 16 massages). Intervention participants received myofascial massage specific to the breast, chest, and shoulder of the affected side. These massages were developed for this study and included a variety of techniques aimed at reducing pain, inflammation, and tissue sensitivity, while also increasing mobility by breaking up scar tissue. The intervention massages involved the following techniques: skin glide (variable time), j stroking (2-3 minutes), vertical stroking (2-3 minutes), strumming (2-3 minutes), fascial stretch (3-5 minutes), circular friction (1-2 minutes), deep fascial release (3-5 minutes), arm pull (60 seconds 
each arm), side latissimus dorsi stretch (3-5 minutes), and twisting (3 minutes). Variations regarding length of time for each technique were based on tissue response and pliability, as deemed by the two massage therapists, as well as feedback from the patient on how well they were tolerating the treatment. Before each of the 16 massage sessions, the therapist would discuss current pain levels with the patient, as well as check mobility and tissue response. Control participants received relaxation massage, also for 30 minutes, which avoided the affected breast, chest, and shoulder area. These Swedish relaxation massages involved light kneading, stroking, efflueurage (gliding), and friction at 2-3 minutes each, rotating across various parts of the entire body.

\section{Outcomes}

After eight weeks, a study coordinator blinded to group assignment telephoned subjects and readministered the same pain, mobility, and quality-oflife questions that were asked at baseline. Subjects were also asked a qualitative question regarding their experience in the study. The single question asked was, "Is there anything about the massage or study experience that you would like to share?"

\section{Analysis}

We used percentages and standard deviations to describe participant characteristics and questionnaire responses. For each participant, a total pain score was calculated by assigning a value of 0 for none, 1 for mild, 2 for moderate, and 3 for severe, and then summing across all 10 items (possible range 0-30 where 30 is worst). Similarly, a total mobility score was calculated by assigning a value of 0 for no difficulty, 1 for mild difficulty, 2 for moderate difficulty, 3 for severe difficulty, and 4 for unable to do, and then summing across all 10 items (possible range 0-40 where 40 is worst). Quality-of-life scores were transformed to a $0-100$ scale, where 100 is best.

For each participant, we calculated change in pain, mobility, and quality of life as the difference between eight-week and baseline scores. We used the paired $t$ test to examine post- vs. pre-scores separately for intervention participants and for control participants. We used the $t$ test to examine changes in scores among intervention vs. control participants. Because women may have some types of pain (e.g., throbbing), but not others (e.g., shooting), the total pain score may underestimate pain burden. As a result, we also examined the proportion of participants who had moderate or worse pain (i.e., moderate or severe) on at least one item. Similarly, we examined the proportion of participants who had moderate or worse mobility limitations (i.e., moderate, severe, or unable to do) on at least one item. We used the chi-square test to compare these proportions between intervention and control participants. A two-tailed $p$ value of $<.05$ was considered significant. All analyses were conducted using JMP version 12.0 (SAS Institute, Cary, North Carolina).

The study team reviewed all responses to the single qualitative question. The comments were categorized as related to pain, mobility, appearance, any other benefits of massage, and any adverse effects. The study team also selected quotes to illustrate the comments.

\section{RESULTS}

\section{Subject Characteristics}

Participant recruitment took place between September 2014 and April 2015 and was done on a rolling basis until we reached our target of 20 participants. Of 31 women initially screened for eligibility, eight were ineligible due to time since surgery being greater than 18 months and three failed to come to their first appointment at the massage therapy office. The subjects who came to their appointment were randomized into intervention (10) and control (10) groups. One intervention participant dropped out after five weeks and answered the final questionnaire at that time. She stated that she was dropping out because she felt much less pain and better mobility after the 5 sessions and did not think further massages were needed. One more participant was then recruited into the intervention group to meet the 20-person goal for completing all 16 massages. Including the one participant who dropped out $(n=21)$, there were a possible 336 massages to be completed. Of those, 325 massages were completed, resulting in a $97 \%$ overall adherence rate.

At baseline, intervention and control groups were generally similar in demographic and medical characteristics (Table 1). More intervention participants had received physical therapy than control participants, but this difference was not statistically significant. None of the participants had seen a pain management specialist.

\section{Quantitative Analysis}

By the end of the trial, there were substantial improvements in pain, mobility, and overall health among intervention participants, but little change among control participants (Table 2). For example, pain scores significantly decreased among intervention participants (baseline 11.8, final 1.1; $p<.001$ ), but did not significantly change among control participants (baseline 8.0, final 8.4; $p=.31$ ). The change in pain scores was also significantly different between groups $(-10.7$ vs. $+0.4, p<.001)$. Feeling downhearted scores improved within both groups and were not significantly different between groups by the end of the trial.

At baseline, the proportion of intervention and control participants with moderate or worse pain 
Table 1. Characteristics of Study Participants

\begin{tabular}{|c|c|c|c|c|}
\hline & & Intervention & Control & $\begin{array}{c}p \\
\text { value }\end{array}$ \\
\hline \multirow[t]{4}{*}{ Age } & & & & .56 \\
\hline & 21-44 years & $3(27 \%)$ & $2(20 \%)$ & \\
\hline & 45-54 years & $5(46 \%)$ & $3(30 \%)$ & \\
\hline & $55+$ years & $3(27 \%)$ & $5(50 \%)$ & \\
\hline \multirow{4}{*}{$\begin{array}{l}\text { Time Post } \\
\text { Surgery }\end{array}$} & & & & .69 \\
\hline & 3-4 months & $3(27 \%)$ & $3(30 \%)$ & \\
\hline & 5-9 months & $4(36 \%)$ & $2(20 \%)$ & \\
\hline & $10-18$ months & $4(36 \%)$ & $5(50 \%)$ & \\
\hline \multirow[t]{4}{*}{ Type of Surgery } & & & & .45 \\
\hline & $\begin{array}{c}\text { Simple } \\
\text { Mastectomy }\end{array}$ & $8(73 \%)$ & $7(70 \%)$ & \\
\hline & Partial Mastectomy & $1(9 \%)$ & $0(0 \%)$ & \\
\hline & $\begin{array}{l}\text { Mastectomy and } \\
\text { Axillary Node } \\
\text { Dissection }\end{array}$ & $2(18 \%)$ & $3(30 \%)$ & \\
\hline \multirow[t]{3}{*}{$\begin{array}{l}\text { Received } \\
\text { Radiation }\end{array}$} & & & & .53 \\
\hline & Yes & $7(64 \%)$ & $5(50 \%)$ & \\
\hline & No & $4(36 \%)$ & $5(50 \%)$ & \\
\hline \multirow[t]{3}{*}{$\begin{array}{l}\text { Received } \\
\text { Physical } \\
\text { Therapy }\end{array}$} & & & & .70 \\
\hline & Yes & $8(73 \%)$ & $2(20 \%)$ & \\
\hline & No & $3(27 \%)$ & $8(80 \%)$ & \\
\hline
\end{tabular}

on at least one item was similar ( $82 \%$ vs. $80 \%, p=$ $.92)$. By the end of the trial, these proportions were significantly better among intervention participants ( $9 \%$ vs. $80 \%, p<.001)$. At baseline, the proportion of intervention and control participants with moderate or worse mobility limitations on at least one item was similar (91\% vs. $80 \%, p=.47)$. By the end of the trial, these proportions were significantly better among intervention participants (11\% vs. $70 \%, p=.003)$.

\section{Qualitative Analysis}

When asked about their overall study experience, all intervention participants reported that their pain and mobility issues improved. One participant said, "I had no idea how much pain I was in...all the time. I feel like I have my life back." Another stated, "This was a life-changing experience, I thought that surviving cancer meant I should be happy regardless of if I couldn't move my arm how I used to. Now I can have it all." Another noted, "I didn't even realize all of the things I was avoiding doing because of the pain and constraint I felt. It's like someone has taken layers of binding off and I can move again!" Intervention participants also expressed satisfaction with the aesthetic effects that myofascial release had on the scar tissue stating "my breast is in the right spot" and "I feel like a woman again."

All control participants reported that the study and receiving massage was a positive experience. Reduction of stress and better sleep were reported as beneficial effects of taking part in the study. One control participant said, "My stress level has gone down so much since receiving the massages. I feel so much better about myself and more in tuned to my body." Another noted, "I haven't slept this good since before I was first diagnosed with breast cancer."

\section{DISCUSSION}

We found that myofascial massage significantly reduced self-reported pain and mobility limitations. Myofascial massage also resulted in significant improvements in self-reported overall health. Both the myofascial massage and the relaxation massage groups had decreased feelings of downheartedness, suggesting that both types of massage had some quality-of-life benefits. Participants tolerated both

Table 2. Pain, Mobility and Quality-of-Life Outcomes

\begin{tabular}{|c|c|c|c|c|c|c|c|c|c|c|}
\hline & \multicolumn{4}{|c|}{ Intervention } & \multicolumn{4}{|c|}{ Control } & \multicolumn{2}{|c|}{$\begin{array}{l}\text { Intervention vs. } \\
\text { Control Change }\end{array}$} \\
\hline & Baseline & Final & Change & $p$ value & Baseline & Final & Change & Pvalue & Difference & $p$ \\
\hline Pain $^{a}$ & 11.8 & 1.1 & -10.7 & $<.001$ & 8.0 & 8.4 & +0.4 & .31 & -11.3 & $<.001$ \\
\hline Mobility $^{b}$ & 16.3 & 1.7 & -14.5 & $<.001$ & 13.2 & 12.4 & -0.8 & .49 & -13.7 & $<.001$ \\
\hline Social Activities ${ }^{c}$ & 87.2 & 94.5 & +7.3 & .27 & 74.0 & 78.0 & +4.0 & .64 & +3.3 & 0.76 \\
\hline Downhearted ${ }^{c}$ & 74.5 & 94.5 & +20.0 & $<.001$ & 68.0 & 86.0 & +18.0 & $<.001$ & +2.0 & 0.74 \\
\hline Overall Health ${ }^{c}$ & 52.3 & 81.8 & +29.5 & $<.001$ & 57.5 & 55.0 & -2.5 & .73 & +32.0 & .002 \\
\hline
\end{tabular}

apain score had a possible range of $0-30$ where 30 is worst.

${ }^{b}$ Mobility score had a possible range of $0-40$ where 40 is worst.

${ }^{\mathrm{c}}$ The quality-of-life scores had a possible range of $0-100$, where 100 is best. 
types of massage, and qualitative feedback was overwhelmingly positive.

Strengths of our study include well-defined subject selection criteria, a randomized controlled trial design, the use of a control group that received a similar amount of time and attention, and use of validated outcome measures. The high adherence of participants $(97 \%)$ to assigned massage treatments is also worth noting. Reasons for this exceptionally high adherence may be the overall value participants found in the massage treatment, both for the outcomes noted in the intervention group and for the stress relief and other positive effects the control group experienced.

The few previous studies of massage for breast cancer surgery differed from our approach in several critical ways. A French study of 20 women found that mechanical massage improved the skin complications of radiation-induced fibrosis. However, this study did not include an active control group. ${ }^{(13)}$ A German study of 86 women found that back, head, and neck massage reduced physical discomfort and improved mood disturbances. However, the study was not designed to focus on the affected breast, chest, and shoulder areas. ${ }^{(14)}$ A Brazilian study of 131 women found that massage therapy did not enhance the results obtained with exercises alone for shoulder range of motion and ipsilateral upper limb function. It did not report on the impact of massage on pain. ${ }^{(15)}$ An American study of 46 patients found that massage led to reductions in pain, stress, and muscle tension, as well as an increase in relaxation. The massage was provided the day after breast cancer surgery and did not focus on the affected breast, chest, and shoulder areas. In addition, there was no control group. ${ }^{(8)}$

Our findings have implications for patients, physicians, and payers. Several study participants stated that dealing with pain or mobility issues was just the price to pay to be cancer-free. Previous research has also found that there is much underreporting of pain among breast cancer surgery patients. ${ }^{(3)}$ Our findings suggest that women with chronic pain or mobility limitations should consider a trial of myofascial massage. Physicians should refer women with persistent pain or mobility issues for massage treatments. It is possible that massage may enhance the effectiveness of traditional treatments such as pain medication and physical therapy, or become another treatment option for challenging shoulder and chest wall issues. In addition, it is important for health insurance companies to cover the cost of therapeutic massage so massage cost is not a barrier for low- and middleincome women.

Several limitations must be considered in interpreting our results. The number of participants was small, most patients had simple mastectomies (vs. other types of surgery), the study was conducted at a single center, and study outcomes were self-reported. We had no details about the extent and quality of previous treatments, such as physical therapy, which may be a confounding factor. None of our study participants had appreciable amounts of lymphedema. As a result, our results may not apply to pain and mobility limitations that are due to lymphedema.

\section{CONCLUSION}

Myofascial massage is a promising treatment to address chronic pain and mobility limitations following breast cancer surgery. Further work in several areas is needed to confirm and expand on our study findings. First, these findings should be demonstrated in larger and more diverse patient groups in multiple centers with additional therapists. Second, other objective measures (e.g., range of motion, functional ability) and patient-centered outcomes (e.g., scarring, aesthetics) should be studied. Third, we need to understand the impact of massage on long-term outcomes.

\section{ACKNOWLEDGMENTS}

This study was supported in part by grants MD002265 and TR000439 from the National Institutes of Health. We are grateful to the women who participated in this study.

\section{CONFLICT OF INTEREST NOTIFICATION}

The authors declare there are no conflicts of interest.

\section{COPYRIGHT}

Published under the CreativeCommons AttributionNonCommercial-NoDerivs 3.0 License.

\section{REFERENCES}

1. Gartner R, Jensen MB, Nielsen J, Ewertz M, Kroman N, Kehlet $\mathrm{H}$. Prevalence of and factors associated with persistent pain following breast cancer surgery. JAMA. 2009;302(18):1985-1992.

2. Hayes SC, Johansson K, Stout NL, Prosnitz R, Armer JM, Gabram S, et al. Upper-body morbidity after breast cancer: incidence and evidence for evaluation, prevention, and management within a prospective surveillance model of care. Cancer. 2012;118(8):2237-2249.

3. Wallace MS, Wallace AM, Lee J, Dobke MK. Pain after breast surgery: a survey of 282 women. Pain. 1996;66(2-3):195-205.

4. Martin ML, Hernández MA, Avendaño C, Rodriguez F, Martinez H. Manual lymphatic drainage therapy in patients with breast cancer-related lymphoedema. BMC Cancer. 2011;11:94.

5. FitzGerald M, Anderson R, Potts J, Payne CK, Peters, KM, Clemens JO, et al. Randomized multicenter feasibility trial of myofascial physical therapy for the treatment of urological chronic pelvic pain syndromes. J Urol. 2009;182(2):570-580. 
6. Crawford C, Boyd C, Paat CF, Price A, Xenakis L, Yang $\mathrm{E}$, et al. The impact of massage therapy on function in pain populations - a systematic review and meta-analysis of randomized controlled trials: Part I, Patients experiencing pain in the general population. Pain Med. 2006;17(7):1354-1375.

7. Cassileth BR, Vickers AJ. Massage therapy for symptom control: outcome study at a major cancer center. J Pain Symptom Manag. 2004;28(3):244-249.

8. Drackley N, Degnim A, Jakub JW, Cutshall SM, Thomley BS, Brodt JK, et al. Effect of massage therapy for postsurgical mastectomy recipients. Clin J Oncol Nurs. 2012;16(2): 121-124.

9. Khan SA, Apkarian AV. The characteristics of cyclical and non-cyclical mastalgia: a prospective study using a modified McGill Pain Questionnaire. Breast Cancer Res Treat. 2002;75(2):147-157.

10. Wingate L. Efficacy of physical therapy for patients who have undergone mastectomies: a prospective study. Phys Ther. 1985;65(6):896-900.

11. Ware JE, Kosinski MM, Keller SD. A 12-item short-form health survey: construction of scales and preliminary validity. Med Care. 1996;34(3):220-233.
12. Listing M, Reisshauer A, Krohn M, Voigt B, Tjahono G, Becker J, et al. Massage therapy reduces physical discomfort and improves mood disturbances in women with breast cancer. Psychooncol. 2009;18(12):1290-1299.

13. Bourgeois JF, Gourgou S, Kramar A, Lagarde JM, Guillot B. A randomized, prospective study using the LPG technique in treating radiation-induced skin fibrosis: clinical and profilometric analysis. Skin Res Technol. 2008;14(1):71-76.

14. Megens A, Harris SR. Physical therapist management of lymphedema following treatment for breast cancer: a critical review of effectiveness. Phsy Ther. 1998;78(12):1302-1311.

15. Amaral M, de Oliveira MM, de Oliveira Ferreira N, Guimaraes RV, Sarian LO, Costa Gurgel MS. Manual therapy associated with upper limb exercises vs. exercises alone for shoulder rehabilitation in postoperative breast cancer. Physiother Theory Pract. 2012;28(4):299-306.

Corresponding author: Jacqueline Dolata, MBA, Center for Reducing Health Disparities, Case Western Reserve University, 2500 MetroHealth Dr., Cleveland, OH 44109-1998, USA

E-mail: jdolata@metrohealth.org 